\title{
Reconfigurable Nanostructured Photonic Metamaterials
}

\author{
Jun-Yu Ou ${ }^{1, *}$, Eric Plum ${ }^{1}$, Liudi Jiang ${ }^{2}$ and Nikolay I. Zheludev ${ }^{1}$ \\ ${ }^{1}$ Optoelectronics Research Centre and Centre for Photonic Metamaterials, University of Southampton, SO17 1BJ, UK \\ ${ }^{2}$ School of Engineering Sciences, University of Southampton, Southampton SO17 1BJ, UK \\ *Email: jo2c09@orc.soton.ac.uk, web: http://www.nanophotonics.org.uk/niz/
}

Here we introduce the first mechanically reconfigurable photonic metamaterials with tunable transmission and reflection characteristics provided by nanoscale movements of the components of the metamaterial structure. In the past control of electromagnetic response of metamaterials has only been possible in the terahertz part of the spectrum through micro-electro-mechanically activated motion.

Using a sophisticated nanofabrication process on a multilayered metal-dielectric membrane we fabricated a two-dimensional array of $\mathrm{C}$-shaped plasmonic resonators (meta-molecules). In our reconfigurable metamaterial alternating rows of the meta-molecules are supported by different gold-silicon nitride layered substrates of nanoscale thickness, see Fig. 1. Through the virtue of differential thermal expansion the mutual position of alternating rows can be controlled by temperature: we observed a substantial and reversible change of the metamaterial's transmission by tuning temperature within a 200K range, see Fig.2.

The metamaterial's transmission spectrum shows several transmission resonances in the near infrared, which are sensitive to coupling between the plasmonic resonators and thus the mutual positions of the meta-molecules in neighboring rows, see Fig. 2. It illustrates the transmission change relative to a reference temperature of $270 \mathrm{~K}$. At the resonance frequencies the metamaterial transmission decreases by up to $35 \%$ when the sample is cooled to 76K. This large temperature-controlled change in the structure's transmission characteristics is reversible by heating the metamaterial back to its initial temperature

We discuss possible improvements and limits of the technology and potential applications of mechanically reconfigurable photonic metamaterials.
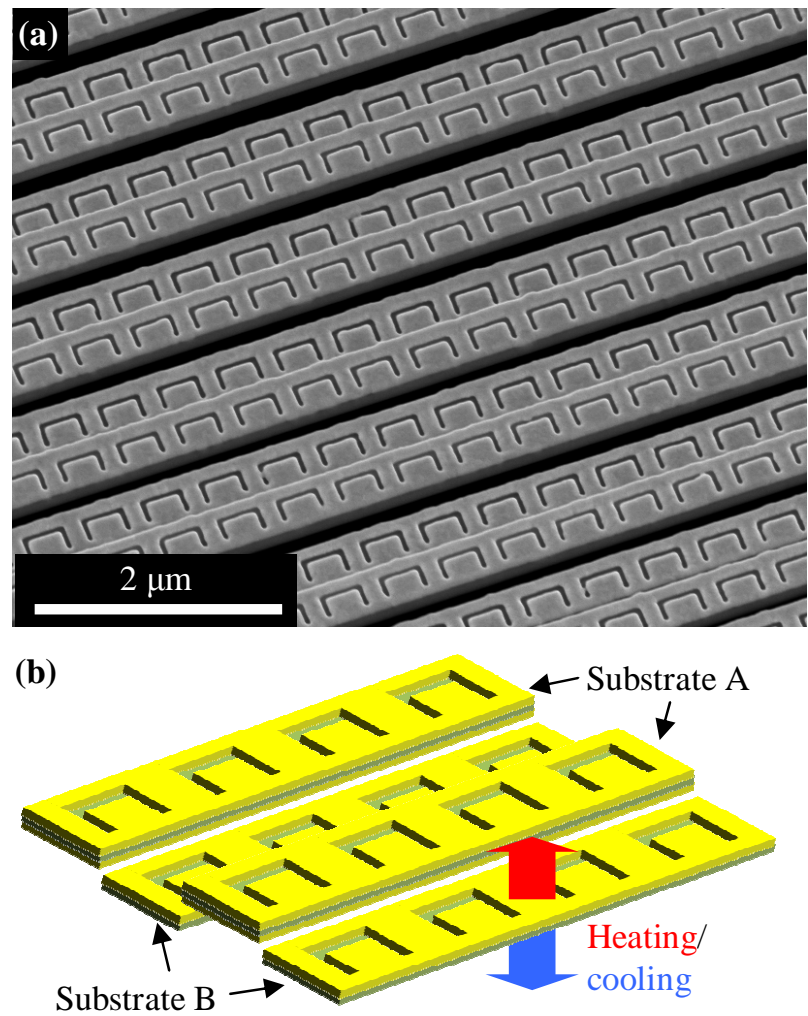

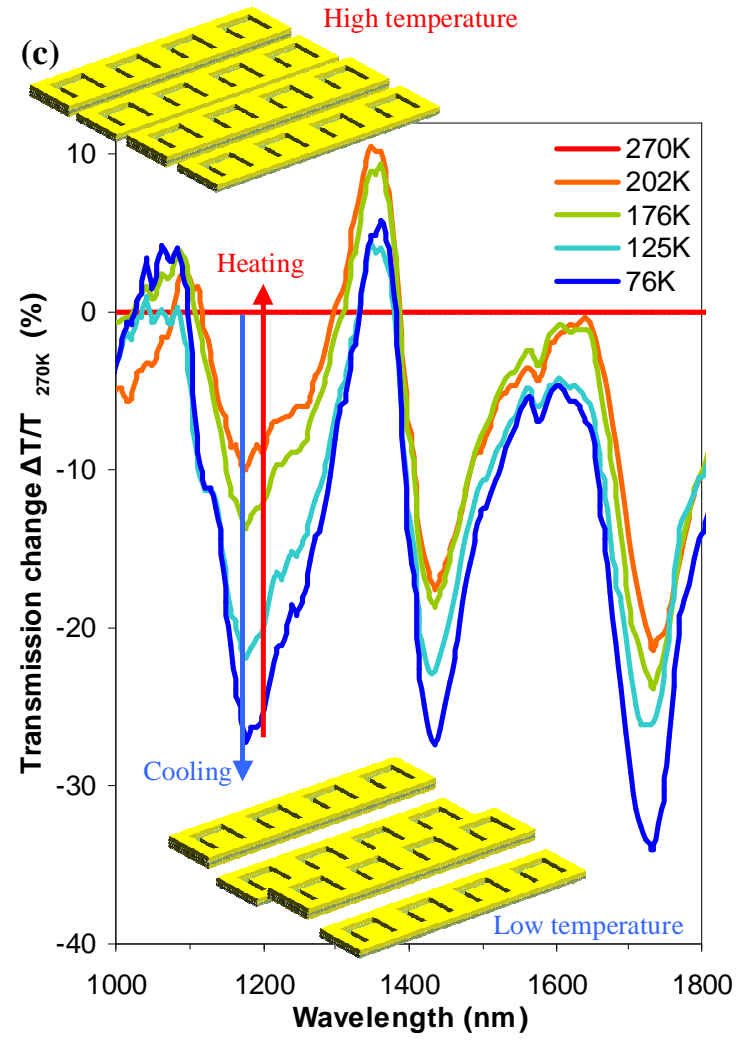

Fig. 1 (a) Scanning electron micrograph of a reconfigurable metamaterial. (b) Schematic of the metamaterial design. (c) Temperature dependence of the transmission spectrum relative to a reference temperature of $270 \mathrm{~K}$. Insets show sketches of the metamaterial at high and low temperatures. 